\title{
Adaptive model and model selection for long-term transmembrane pressure prediction in membrane bioreactors
}

\author{
H. Oishi, H. Kaneko, K. Funatsu* \\ Department of Chemical System Engineering, The University of Tokyo, Hongo 7-3-1, Bunkyo-ku, Tokyo
} 113-8656, Japan

* Corresponding author. Tel: +81-3-5841-7751

Hongo 7-3-1, Bunkyo-ku, Tokyo 113-8656, Japan

e-mail: funatsu@chemsys.t.u-tokyo.ac.jp

\begin{abstract}
Fouling is one of the most significant problems in membrane bioreactors (MBRs). Membranes must be washed with chemicals at appropriate times before severe fouling occurs. Long-term transmembrane pressure (TMP) prediction is attempted to plan the schedule for chemical cleaning. TMP is directly proportional to membrane resistance caused by fouling under the condition of constant-rate filtration. Statistical models have previously been used to predict TMP from operating parameters in MBRs. However, TMP predictions are difficult when operating conditions or water quality varies. We therefore propose to introduce adaptation and selection mechanisms to statistical models. Multiple TMP prediction models are updated with new measurements and a target model is selected, based on the predictive ability of the models, for long-term TMP prediction. Through case studies using two data sets obtained from actual MBRs, we confirmed that the performance of long-term TMP prediction was improved by using the proposed method because the models can adapt appropriately to changes in operating conditions or water quality.
\end{abstract}

\section{Keywords}

fouling prediction; membrane resistance; long-term transmembrane pressure prediction; statistical model; cleaning schedule 


\section{Introduction}

A membrane bioreactor (MBR) [1] is a water treatment process that uses membranes to remove activated sludge from treated water. In a traditional water treatment process, i.e. sedimentation, separation is performed using gravity. MBRs offer advantages such as space-saving and short treatment times compared with gravity sedimentation.

One of the most significant problems in MBRs is membrane fouling [2], a phenomenon whereby foulants such as activated sludge and colloids accumulate on the membranes and block the membrane pores. As fouling progresses, for example during constant-rate operation, the transmembrane pressure (TMP) required for separation increases and operational costs increase.

To reduce fouling, physical cleaning such as aeration and backwashing is performed during the operating cycle, but physical cleaning cannot completely remove foulants. Therefore, it is necessary to clean membranes using chemicals, named chemical cleaning, at appropriate times. Because preparation takes about one week, it is important to have advance knowledge of the most appropriate time for chemical cleaning. Long-term TMP predictions are indispensable for planning the schedule of chemical cleaning. TMP is directly proportional to membrane resistance, which increases through fouling under constant-rate filtration conditions.

Kaneko and Funatsu developed a TMP jump (a rapid increase in TMP) prediction model [3]. Using MBR parameters such as operating conditions and water quality, a statistical model discriminates data before and after TMP jumps. Karabelas and Sioutopoulos modeled the progression of fouling under conditions of constant-rate and constant-pressure filtration [4] and found that the specific resistance $(\alpha)$ is an important factor related to fouling. However, they did not have sufficient data available to predict TMP. Kim et al. modeled four different fouling mechanisms and proposed a TMP prediction model in which these mechanisms were combined [5]. Predictive accuracy for TMP was improved by combining four models and the most dominant fouling mechanism was identified. However, there were large errors for long-term TMP prediction.

Sung et al. developed a membrane resistance $(\mathrm{R})$ prediction model and used this for a long-term TMP prediction method [6]. At time, $t$, the relationship between $T M P_{(t)}$ and $R_{(t)}$, which is the membrane resistance caused by fouling, takes the following form:

$$
T M P_{(t)}=\mu_{(t)} J_{(t)} R_{(t)},
$$

where $\mu_{(\mathrm{t})}$ is the viscosity of treated water and $J_{(t)}$ is the flux of treated water (permeate) through membranes. In an $\mathrm{R}$ prediction model, an output variable (objective variable, $\mathbf{y}$-variable) is $R_{(t)}$ and four input variables (explanatory variables, $\mathbf{X}$-variables) are the elapsed time $t, J_{(t)}, n^{\text {th }}$ power of TMP at $t-1$, and the difference in TMP between $t-1$ and $t$. The form of the $\mathrm{R}$ prediction model is given as follows:

$$
R_{(t)}=f\left(t, J_{(t)}, T M P_{(t-1)}^{n}, \Delta T M P_{(t-1)}\right) .
$$

Because set values of $\mathrm{J}$ can be used as an X-variable, TMP can be predicted over the long-term by estimating $R_{(t)}$ using Eq. (2) and transforming $R_{(t)}$ to $T M P_{(t)}$ via Eq. (1), repeatedly. Although four variables are used in Eq. (2), other variables such as operating conditions and water quality can be added to the $\mathbf{X}$-variables when the measurement frequencies of these variables are the same as for J and TMP. By adding to the $\mathbf{X}$-variables, variables that are thought to contribute to fouling can be considered. However, it must be noted that future values are required during long-term TMP prediction. In these cases, operating conditions and water quality will either be 
assumed to be constant or future values must be predicted.

Using the method of Sung et al., model construction accuracy was high but the errors in long-term TMP prediction were large. An R prediction model was constructed using data obtained in the past and TMP was predicted with the constructed model. Although the model was used for an extended period of time, its accuracy for long-term TMP prediction was not considered when the model was constructed and parameters used in the regression models were optimized considering the accuracy of Eq. (2). However, there is a difference between a model having high accuracy in model construction and a model having high performance for long-term TMP prediction. In addition, the physical states in MBRs, operating conditions and water quality change with time and accordingly the relationships between variables also change with time. Because an $\mathrm{R}$ prediction model cannot adapt to changes in these relationships, the accuracy of the model decreases with time.

We therefore seek to improve the performance for long-term TMP prediction and propose to update an $\mathrm{R}$ prediction model using the latest measurements. As a method of updating the models, we apply a moving window (MW) technique that is widely used in the soft sensor field [7]. In chemical and industrial plants, soft sensors are widely used to predict $\mathbf{y}$-variables that are difficult to measure, such as the concentration and density of products, using $\mathbf{X}$-variables that are easy to measure, such as temperature and pressure. The MW model is known as a model that can adapt to the degradation of soft sensors' predictive accuracy with time [8]. Regression models will also adapt to changes in MBR conditions by updating the models and thus the accuracy of long-term TMP prediction will improve.

In an MW technique, when $m$ is the number of training data used for model construction, differences in $m$ generate different models. Because the prediction accuracy of these models would change with time, it is necessary to select an appropriate model for each time. Therefore, we propose a model selection method based on the accuracy of long-term TMP prediction. The trade-off of inclusion of many flux variations in training data to predict the flux variations and adaptation to the new state in MBRs is handled by the proposed model selection.

This paper is organized as follows. First, in Section 2, we explain the MW R prediction model and the model selection method. To verify the effectiveness of our proposed method, we present case studies using actual MBR data in Section 3. Finally, we conclude the paper in Section 4.

\section{Method}

\subsection{MW R prediction model}

In previous research on $\mathrm{R}$ prediction models, TMP and $\triangle \mathrm{TMP}$ were used as X-variables [6]. According to Eq. (1), TMP changes when $\mathrm{J}$ changes, although $\mathrm{R}$ remains constant. When $\mathrm{J}$ changes frequently, the correlation between $\mathrm{J}$ and TMP is accordingly high, and an R prediction model becomes sensitive to variation in J. For this reason, $R_{(t-1)}$ and $\Delta R_{(t-1)}$ are used as $\mathrm{X}$-variables in the $\mathrm{R}$ prediction model instead of $T M P_{(t-1)}$ and $\Delta T M P_{(t-1)}$. The equation for the $\mathrm{R}$ prediction model in this study is given as follows:

$$
R_{(t)}=f\left(t, J_{(t)}, R_{(t-1)}^{n}, \Delta R_{(t-1)}\right) .
$$

The $\mathrm{R}$ prediction model is updated when MBR data are measured and predicts TMP over the long-term. The logic flow for model updating and TMP prediction is shown in Fig. 1. For the first MBR operation or immediately after chemical cleaning, $t$ is $1 . T M P_{(t)}$ and $J_{(t)}$ are measured and $R_{(t)}$ is calculated using Eq. (1). If $T M P_{(t)}$ and $J_{(t)}$ are not measured, for reasons of instrument or measurement problems, model updating is skipped and predicted TMP 
values at $t$ are the same as those at $t-1$. After measurement of $T M P_{(t)}$ and $J_{(t)}, m$ data are prepared as training data. $m$ is the number of training data points. If the number of data points is less than $m$, all data are used as training data. An R prediction model is constructed using a statistical regression method. The partial least squares (PLS) method [9], which is a linear regression method, and the support vector regression (SVR) method [10], which is a nonlinear regression method, are the major regression methods used. PLS was used in this study. The details of PLS are shown in Appendix A. The constructed model predicts $R_{(t+1)}, R_{(t+2)}, \ldots, R_{(t+t \max )} \cdot t_{\max }$ is a maximum time of long-term TMP prediction and should be less than the remaining time to chemical cleaning. $R_{(\mathrm{t}+1)}, R_{(t+2)}, \ldots$, $R_{(t+t \max )}$ are estimated using Eq. (3) and transformed to $T M P_{(t+1)}, T M P_{(t+2)}, \ldots, T M P_{(t+t \max )}$ using Eq. (1). After long-term TMP prediction, the value of $t$ is incremented. When chemical cleaning is required, for example, after a TMP value exceeds a certain threshold value, membranes are washed and $t$ is reset to 1 ; otherwise TMP and $\mathrm{J}$ are measured.

\subsection{Model selection}

Differences in $m$ and the kind of $\mathbf{X}$-variables included in Eq. (3) generate different PLS models. The best model, offering high accuracy for long-term TMP prediction, can change with time because of changes in operating conditions and water quality. We propose a model selection method based on long-term TMP prediction accuracy. Models where $m$ differs independently predict the latest TMP values from several hours or several days ago. Root mean squared error (RMSE) values between predicted values and observed values are calculated as the evaluation index. Figure 2 shows an image of model selection when the number of models is two. $n_{\text {sel }}$ is the number of data used for evaluation and $t_{\text {sel }}$ is the length of time applied for long-term TMP prediction. The latest $n_{\text {sel }}$ TMP values are predicted from $t_{\text {sel }}$ times before. TMP values are predicted from $t-t_{\mathrm{sel}}$ to $t$, from $t-t_{\mathrm{sel}}-1$ to $t-1$ and from $t-t_{\mathrm{sel}}-2$ $\left(t-t_{\mathrm{sel}}-n_{\mathrm{sel}}+1\right)$ to $t-2\left(t-n_{\mathrm{sel}}+1\right)$. RMSE values are calculated between $n_{\text {sel }}$ predicted values and $n_{\text {sel }}$ observed values. The precision of long-term TMP prediction will be high when the value of RMSE value is low, so a model which has the lowest RMSE value is selected. Because the RMSE value of model 1 is lower than that of model 2, TMP values predicted with model 1 are selected as the predicted TMP values from $t$.

The equation itself of our final adaptive model is the same as Eq. (3), but the parameters in Eq. (3) adaptively change using new measurements to fit to the changes in operating conditions or water quality in MBRs.

The modeling of flux variations is also important and has two meanings; to model past change of flux and to predict future change of flux. If training data in MW modelling include flux variations, we can model the flux variations and predict the same type of future change of flux by using the proposed method, since our proposed model is a data-based model. Therefore, it is important to include many types of flux change in training data. However, only the recent data should be used as training data when the states in MBRs are changing rapidly and old data have a bad effect to the model's predictive performance. To model many flux variations and to adapt the model to the recent state in an MBR are trade-off. We handle this trade-off in the proposed model selection, monitoring the performance of MW models whose $m$ is different and selecting appropriate $m$ each time in the prediction procedure.

\section{Results and discussion}

\subsection{Data}

We analyzed two data sets obtained from different actual MBRs using the proposed method. 


\section{MBR-A}

MBR-A was a pilot scale MBR, comprising anoxic and aerobic tanks. A membrane module was immersed in the aerobic tank. Over the entire operation, we used the data taken under constant-rate operating conditions. We used data in five zones. A zone means from the previous chemical cleaning to the next one. We used data in the first three zones as a training data set and data in the last two zones as a test data set.

\section{MBR-B}

MBR-B was operated in an actual sewage treatment facility and comprised anaerobic, anoxic and aerobic tanks. A membrane module was immersed in the aerobic tank. $\mathrm{J}$ frequently changed in MBR-B and thirteen zones were used. We used data in the first six zones as a training data set and data in the last seven zones as a test data set.

Because R values suffer noise, we applied Savitzky-Golay filtering [11, 12]. Also, because normally $\mathrm{R}$ increases with time, the approximate function was set as a monotonically increasing function. The order of the function was two and the number of points used for fitting was 201. After smoothing, R and TMP were preprocessed, and the minimum values of $\mathrm{R}$ and TMP were zero in each zone.

\subsection{Prediction results}

Here, we compare a previous method with the proposed method. In the previous method, the R prediction model was constructed using the training data set and the TMP for the test data set was predicted. In the proposed method, $m$ was set as 100, 200, .,, 1000 for MBR-A and 100, 200, .,, 1500 for MBR-B. Data where TMP exceeded $15 \mathrm{kPa}$ were not used for model construction. $n$, which is the multiplier of $\mathrm{R}$ was set to 1 . $n_{\text {sel }}$ for MBR-A was 6 and $n_{\text {sel }}$ for MBR-B was 3. $t_{\text {sel }}$ was 24 hours (one day). $t_{\max }$ was 168 hours (one week). In addition, when the number of values for which $\triangle$ TMP was less than 0 exceeded a threshold value, a penalty value (100) was added to RMSE because TMP normally increases with time. The threshold value was $t_{\max } / 3$, because almost all models were assigned a penalty when the threshold value was too low. The parameters $n_{\text {sel }}, t_{\text {sel }}$ and $t_{\text {max }}$ did not affect the results significantly.

The results of long-term TMP prediction for MBR-A and MBR-B are shown in Figs. 3 and 4, respectively. Observed values from 1 to $t$ and predicted values and observed values from $t$ to $t+t_{\max }$ are shown. At any time, the accuracies of long-term TMP prediction with the proposed method were higher than that with the previous method Referring to Fig. 3(a) and 3(c), the R prediction model could not predict the change in slope for TMP caused by changes in operating conditions and water quality and therefore predicted TMP values increased. For Fig. 3(b) and 3(c), the MW R prediction model could predict the slope change for TMP and could therefore adapt to these changes by updating the model. In Fig. 3(e), the R prediction model was not able to predict the rapid increase in TMP, i.e. a TMP jump, and in Fig. 4(a) and 4(c), the predicted TMP values increase excessively. These trends that the $\mathrm{R}$ prediction model could not predict TMP accurately were also seen at times before and after. However, using the proposed method, models could adapt to the changes in operating conditions and water quality, and thus, a TMP jump in Fig. 3(f) and the gradual increase in TMP in Fig. 4(b) and 4(d) were predicted with high accuracy. In Zone 7 of the MBR-B, because J decreased suddenly at around 210 hours, the observed TMP values were correspondingly low in Fig. 4(a) and 4(b). Although the R values predicted with the previous method did not decrease, the predicted TMP values in Fig. 4(a) decreased suddenly because J was used to transform R to TMP. However, R values predicted with the proposed method increased significantly when $\mathrm{J}$ decreased, and even after transforming R to TMP, the TMP values predicted with the proposed method were high. The increase in predicted 
$\mathrm{R}$ values was caused by the negative regression coefficient for $\mathrm{J}$. Because the variation in $\mathrm{J}$ between $t$ and $t+t_{\max }$ was significantly different from that used in the training data, errors in $\mathrm{R}$ became large when $\mathrm{J}$ varied. Future changes in $\mathrm{J}$ should therefore be considered in model construction and model selection.

In Fig. 4(e) and 4(f), TMP decreased sharply because $\mathrm{J}$ decreased. The traditional R prediction model could not adapt to this decrease in $\mathbf{J}$ so the predicted TMP values did not increase markedly, as shown in Fig. 4(e). In contrast, the MW R prediction model could adapt to the change in $J$ and therefore predicted TMP values were in good agreement with observed TMP values.

When the threshold in the TMP value for chemical cleaning was set to $15 \mathrm{kPa}$, the timing of chemical cleaning was between $t$ and $t+t_{\max }$ in Figs. 3(e), 3(f), 4(e) and 4(f). Because TMP values predicted with the traditional method did not exceed $15 \mathrm{kPa}$, the schedule of chemical cleaning could not be planned in Fig. 3(e) and 4(e). However, the accuracy of the proposed method for long-term TMP prediction was high and therefore the timing of chemical cleaning could be predicted almost 100 hours ahead of time (Fig. 3(f) and 4(f)). We thus confirmed that the timing of chemical cleaning can be accurately predicted using the proposed MW R prediction model.

The RMSE values of long-term TMP predictions with the simple MW R prediction models whose $m$ is 100 or 1000 (constant) and the RMSE values in Fig. 3 and Fig. 4 are shown in Tables 1 and 2, respectively. The RMSE values were calculated between observed TMP values and predicted TMP values from $t+1$ to $t+t_{\max }$. In Tables 1 and 2, all RMSE values of the proposed MW R prediction models in which MW models are selected in the prediction procedure are lower than those of the traditional ones. However, RMSE values calculated with the simple MW R prediction models are sometimes higher than those of the traditional ones. For example, the model whose $m$ is 1000 in MBR-A, Zone 15, $t$ is 351 . The simple MW R prediction model cannot adapt to the changes in operating conditions or water qualities, which means that it is difficult to determine the optimal window size while the states in MBRs are changing. Even in this situation, the predictive model can be selected using the proposed method. The effectiveness of the model selection method was confirmed.

\section{Conclusion}

We have developed an MW R prediction model that is updated with new measurement data and a model selection method based on the predictive ability of the models. Case studies using data obtained from actual MBRs were used to demonstrate that the accuracy of long-term TMP prediction was improved by using an MW R prediction model and selecting appropriate models. Because an MW R prediction model can adapt to the change in operating conditions and water quality, TMP values predicted with the proposed method were closer to observed values than those predicted using the traditional method in various situations. Our proposed method is effective for scheduling chemical cleaning of MBRs. However, errors in predicted TMP values were large when $\mathbf{J}$ changed during long-term TMP prediction. Therefore, the consideration of future changes in J is required in model construction and model selection.

\section{Acknowledgements}

The authors acknowledge the support of the Core Research for Evolutionary Science and Technology (CREST) project 'Application of Integrated Intelligent Satellite System (IISS) to construct regional water resources utilization system' of the Japan Science and Technology Agency (JST).

\section{References}

[1] F. Meng, S.R. Chae, M. Kraume, H.S. Shin, F. Yang, Recent advances in membrane bioreactors (MBRs): 
Membrane fouling and membrane material, Water Res. 43 (2009) 1489-1512.

[2] M. Kraume, D. Wedi, J. Schaller, V. Iversen, A. Drews, Fouling in MBR: What use are lab investigations for full scale operation?, Desalination 236 (2009) 94-103.

[3] H. Kaneko, K. Funatsu, Visualization of Models Predicting Transmembrane Pressure Jump for Membrane Bioreactor. Ind. Eng. Chem. Res. 51 (2012) 9679-9686.

[4] A.J. Karabelas, D.C. Sioutopoulos, Toward improvement of methods for predicting fouling of desalination membranes - The effect of permeate flux on specific fouling resistance, Desalination 343 (2014) 97-105.

[5] M. Kim, B. Sankararao, S. Lee, C.K. Yoo, Prediction and Identification of Membrane Fouling Mechanism in a Membrane Bioreactor Using a Combined Mechanistic Model, Ind. Eng. Chem. Res. 52 (2013) 17198-17205.

[6] K. Sung, H. Kaneko, K. Funatsu, Construction of Long-Term Transmembrane Pressure Estimation Model for a Membrane Bioreactor, J. Comput. Aided Chem. 13 (2012) 10-19. [Japanese]

[7] H. Kaneko, M. Arakawa, K. Funatsu, Development of a New Soft Sensor Method Using Independent Component Analysis and Partial Least Squares, AIChE Journal 55 (2009) 87-98.

[8] H. Kaneko, K. Funatsu, Classification of the Degradation of Soft Sensor Models and Discussion on Adaptive Models, AIChE J. 59 (2013) 2339-2347.

[9] S. Wold, M. Sjöström, L. Eriksson, PLS-regression: a basic tool of chemometrics, Chemomtr. Intell. Lab. Syst. 58 (2001) 109-130.

[10] C.M. Bishop, Pattern recognition and machine learning, Springer, New York, 2006, pp. 325-345.

[11] N. Yoshimura, M. Takayanagi, Chemometrics Calculations with Microsoft Excel (5) - Smoothing and Numerical Differentiation, J. Comput. Chem. Jpn. 11 (2012) 149-158. [Japanese]

[12] A. Savitzky, M.J.E. Golay, Smoothing and Differentiation of Data by Simplified Least Squares Procedures, Anal. Chem. 36 (1964) 1627-1639. 


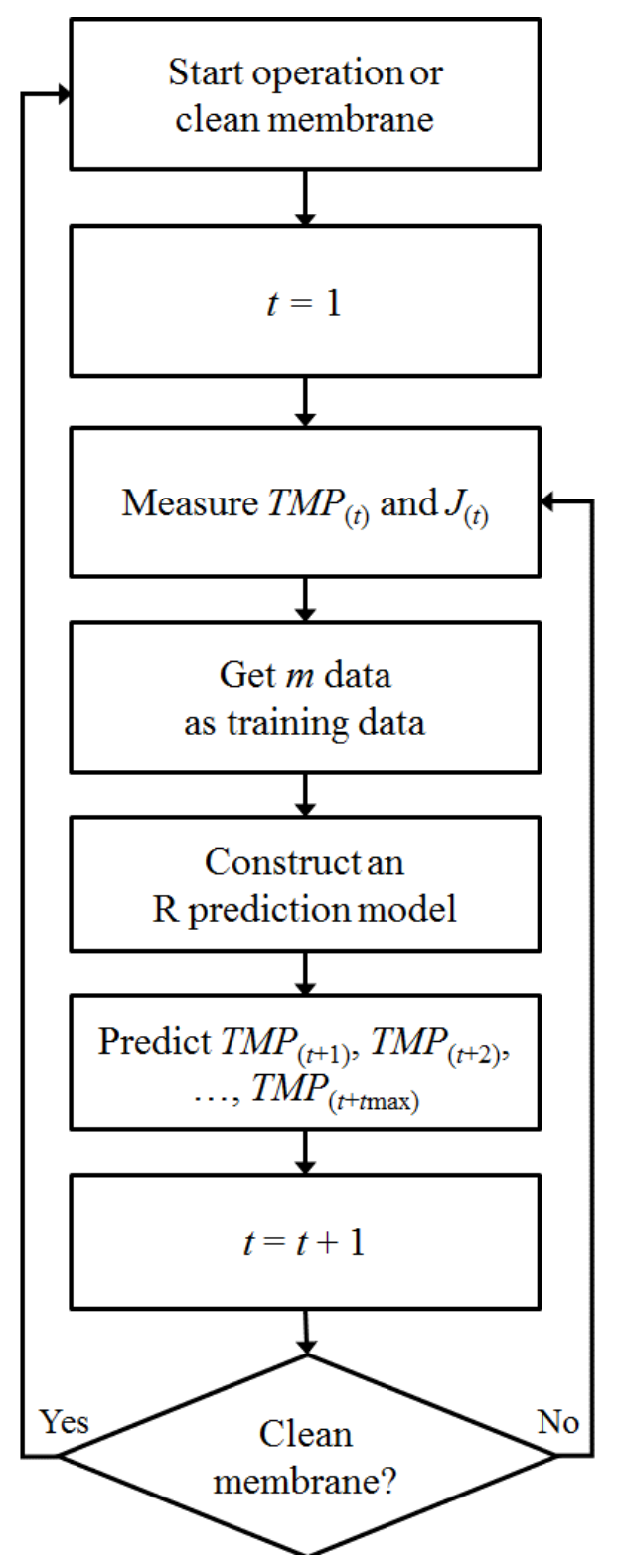

Fig. 1. Flow of model update and TMP prediction using an MW R prediction model. 


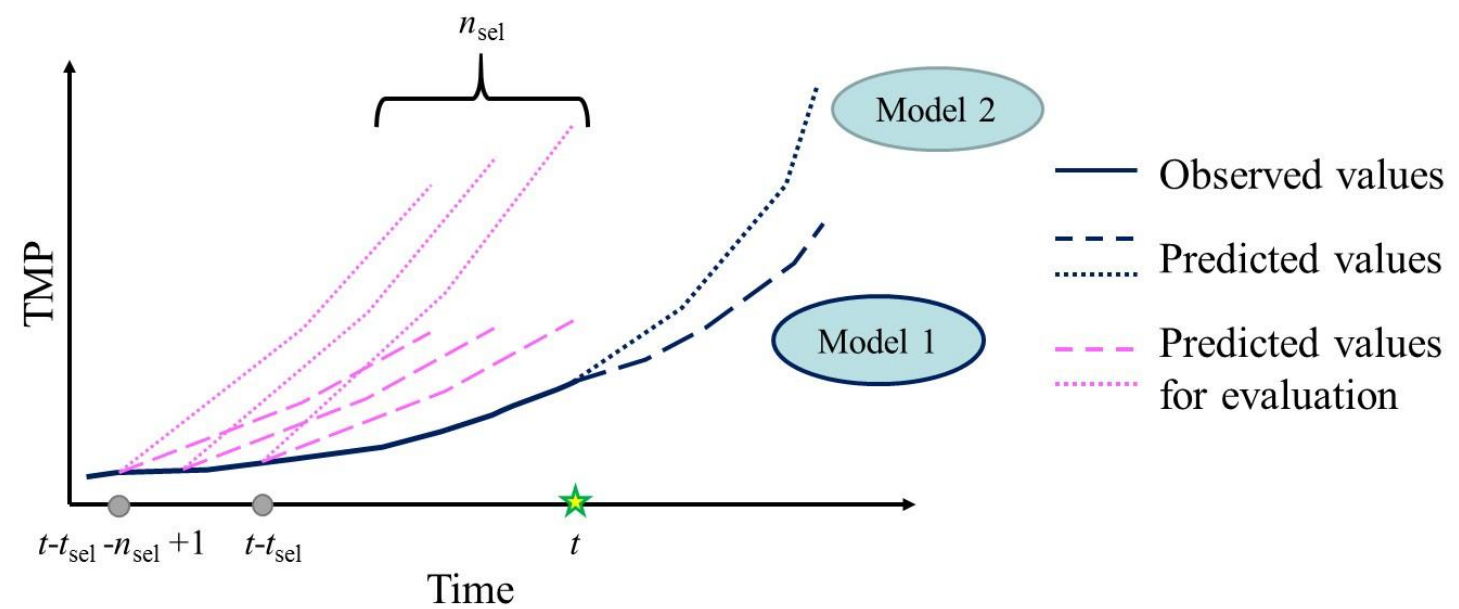

Fig. 2. Image of model selection when the number of models is two $\left(n_{\text {sel }}=3\right)$.

The broken line and the dotted line are values predicted with model 1 and model 2, respectively. 


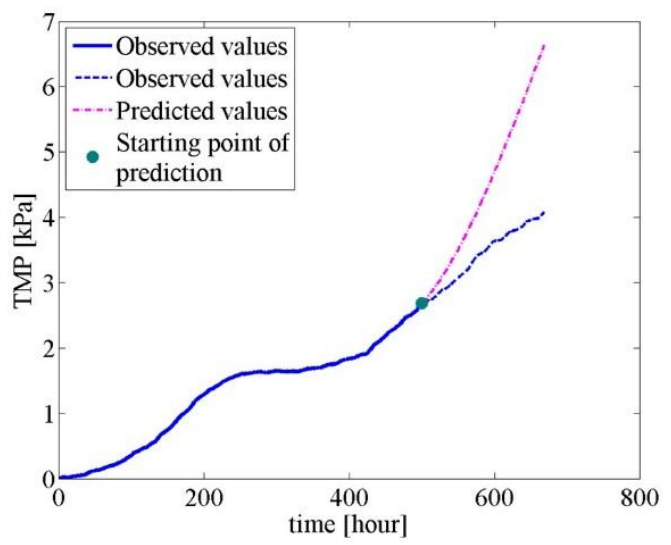

(a) Zone 4, $t=500$,

$\mathrm{R}$ prediction model

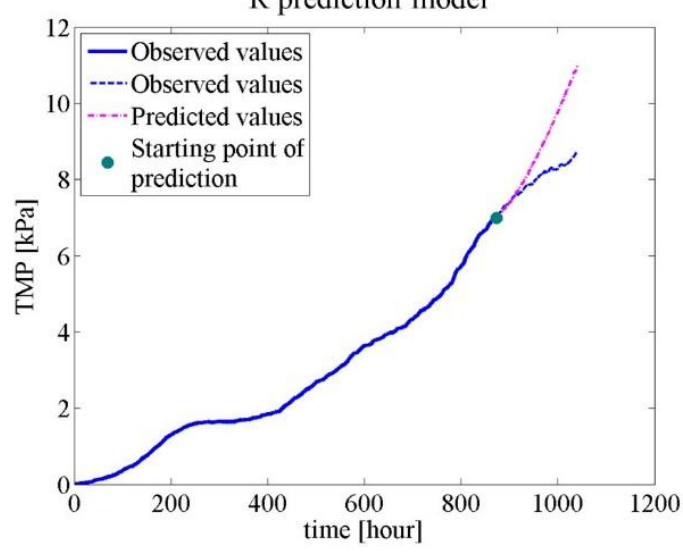

(c) Zone 4, $t=873$,

$\mathrm{R}$ prediction model

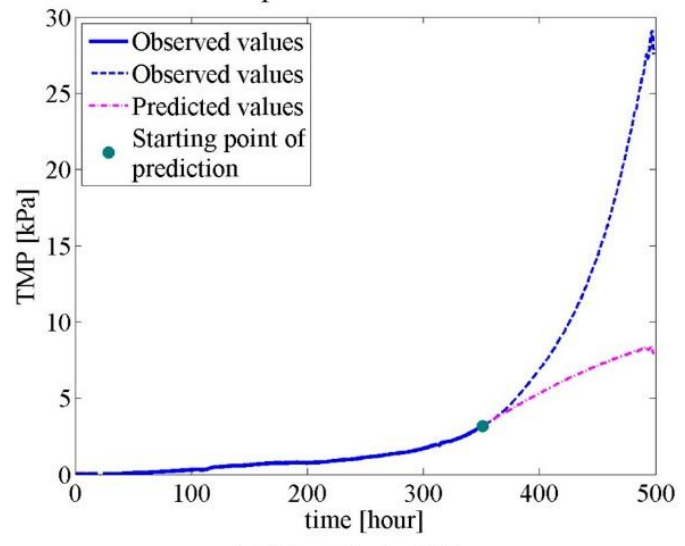

(e) Zone 5, $t=351$,

$\mathrm{R}$ prediction model

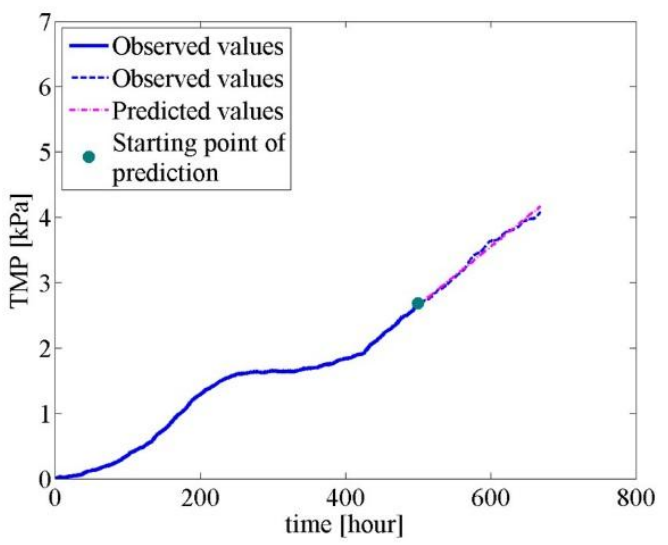

(b) Zone 4, $t=500$,

MW R prediction model

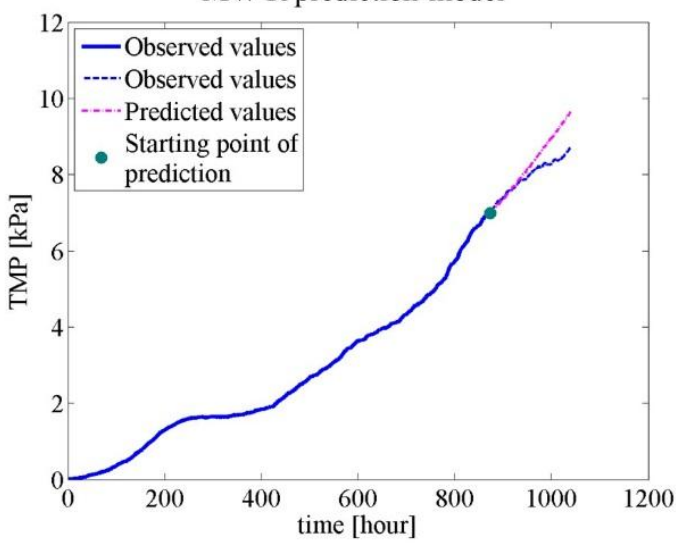

(d) Zone 4, $t=873$,

MW R prediction model

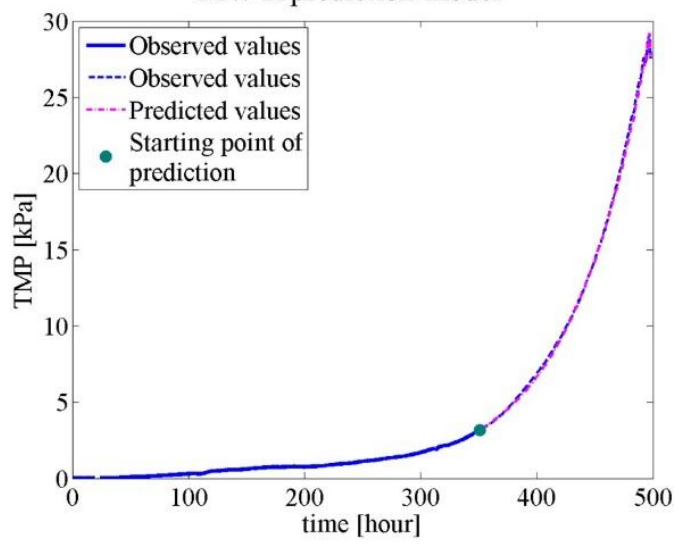

(f) Zone 5, $t=351$,

MW R prediction model

Fig. 3. Results of long-term TMP prediction for MBR-A 


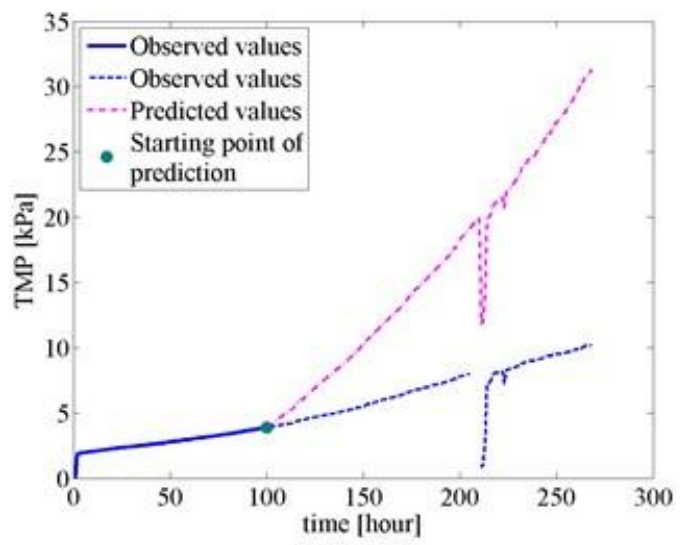

(a) Zone $7, t=100$,

$\mathrm{R}$ prediction model

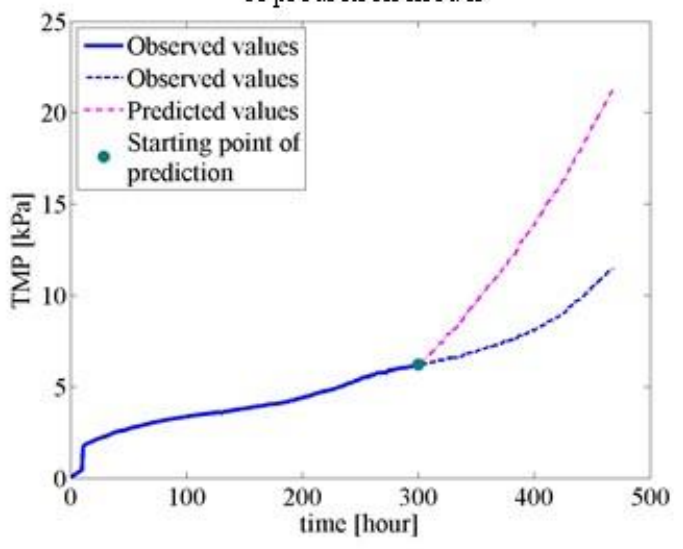

(c) Zone 10, $t=300$,

$R$ prediction model

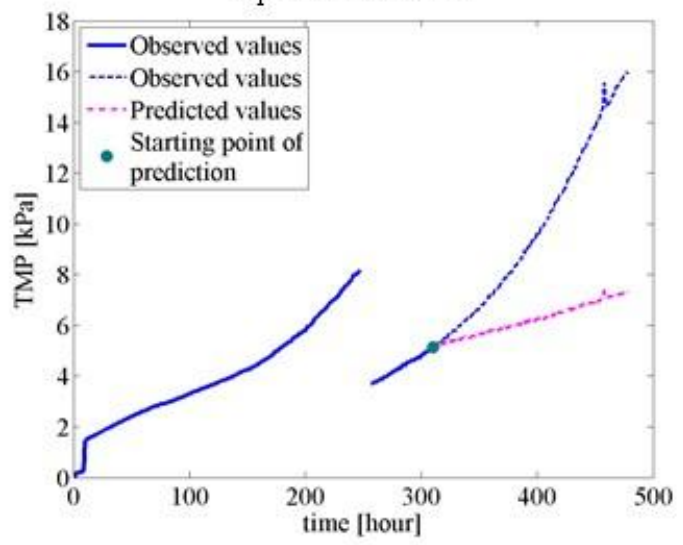

(e) Zone $11, t=310$,

R prediction model

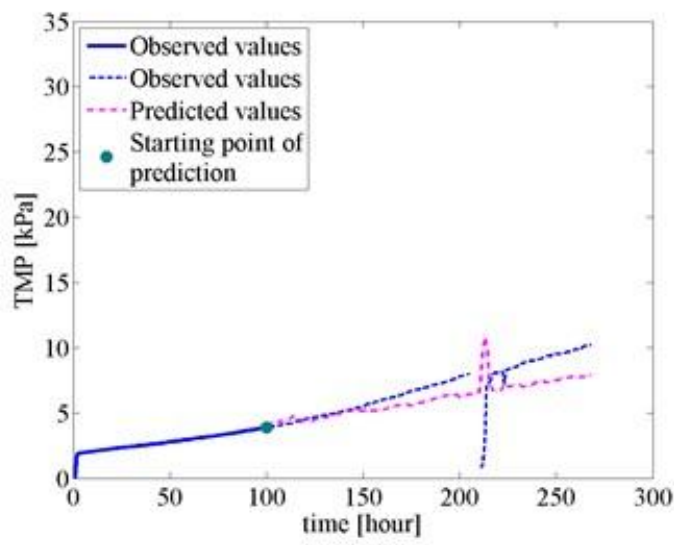

(b) Zone $7, t=100$,

MW R prediction model

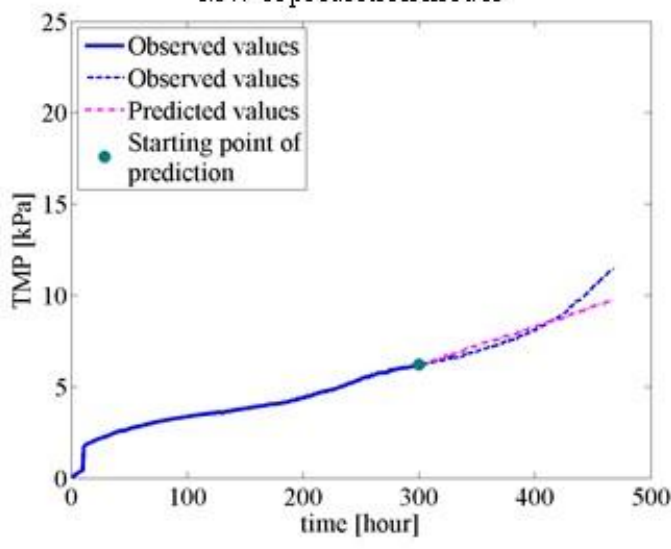

(d) Zone 10, $t=300$,

MW R prediction model

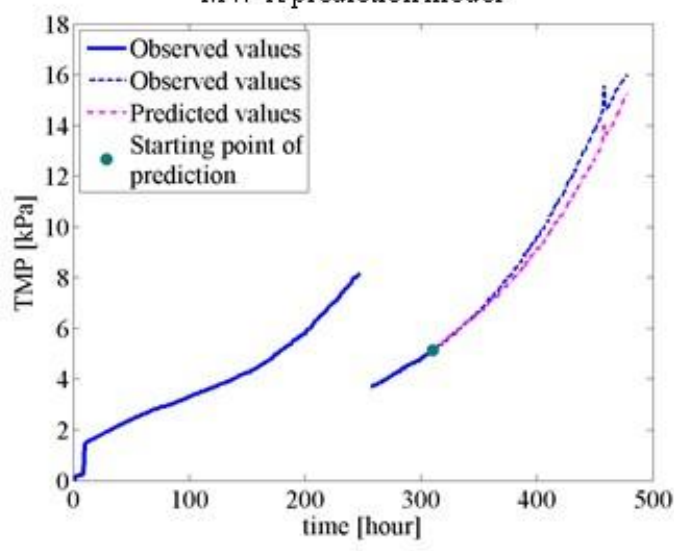

(f) Zone $11, t=310$,

MW R prediction model

Fig. 4. Results of long-term TMP prediction for MBR-B 
Table 1. RMSE values of long-term TMP prediction with each model for MBR-A

\begin{tabular}{cccccc}
\hline \multirow{2}{*}{ Zone } & \multirow{2}{*}{$t$} & R prediction model & \multicolumn{3}{c}{ MW R prediction model } \\
& & & Proposed & $m$ is 100 & $m$ is 1000 \\
\hline 12 & 500 & 1.256 & 0.078 & 0.078 & 0.456 \\
12 & 873 & 1.103 & 0.478 & 0.841 & 0.437 \\
15 & 351 & 8.453 & 0.198 & 0.198 & 9.949 \\
\hline
\end{tabular}


Table 2. RMSE values of long-term TMP prediction with each model for MBR-B

\begin{tabular}{cccccc}
\hline \multirow{2}{*}{ Zone } & \multirow{2}{*}{$t$} & \multirow{2}{*}{ R prediction model } & \multicolumn{3}{c}{ MW R prediction model } \\
& & & Proposed & $m$ is 100 & $m$ is 1000 \\
\hline 7 & 100 & 10.917 & 1.626 & 1.626 & 4.230 \\
10 & 300 & 5.626 & 0.576 & 7.059 & 9.078 \\
11 & 310 & 4.470 & 0.641 & 0.641 & 4.448 \\
\hline
\end{tabular}

